\title{
Development of a Force Amplitude- and Location-Sensing Device Designed to Improve the Ligament Balancing Procedure in TKA
}

\author{
Denis Crottet ${ }^{1,4}$, Thomas Maeder ${ }^{2}$, Daniel Fritschy ${ }^{3}$, Hannes Bleuler ${ }^{4}$, Lutz-P. Nolte ${ }^{1}$, and \\ Ion P. Pappas ${ }^{1}$ \\ 1) M.E. Müller Research Center, University of Bern, 3014 Bern, Switzerland \\ 2) Laboratoire de Production Microtechnique, Ecole Polytechnique Fédérale de Lausanne, 1015 Lausanne, Switzerland \\ 3) Policlinique des Services de Chirurgie, Hôpi- taux Universitaires de Genève, 1205 Geneva, Switzerland \\ 4) Laboratoire de Systèmes Robotiques, Ecole Poly-technique Fédérale de Lausanne, 1015 Lausanne, Switzerland
}

Version of record: IEEE Transactions on Biomedical Engineering 52 (9), 1609-1611, 2005.

http://hdl.handle.net/10.1109/TBME.2005.851504

\begin{abstract}
To improve the ligament balancing procedure during total knee arthroplasty a force-sensing device to intraoperatively measure knee joint forces and moments has been developed. It consists of two sensitive plates, one for each condyle, a tibial base plate and a set of spaces to adapt the device thickness to the patient-specific tibiofemoral gap. Each sensitive plate is equipped with three deformable bridges instrumented with thick-film piezoresistive sensors, which allow accurate measurements of the amplitude and location of the tibiofemoral contact forces. The net varus-valgus moment is then computed to characterize the ligamentous imbalance. The developed device has a measurement range of $0-500 \mathrm{~N}$ and anintrinsicaccuracyof $0.5 \%$ fullscale.Experimentaltrialsonaplastic knee joint model and on a cadaver specimen demonstrated the proper function of the device in situ. The results obtained indicated that the novel force-sensing device has an appropriate range of measurement and a strong potential to offer useful quantitative information and effective assistance during the ligament balancing procedure in total knee arthroplasty.
\end{abstract}

Keywords: Force measurement, ligament balancing, piezoresistive de- vices, total knee arthroplasty

\section{Introduction}

Recent analysis has shown that failure rates of primary total knee arthroplasty (TKA) are $9 \%$ at 10 years, $16 \%$ at 15 years, and $22 \%$ at 20 years [11]. Two common causes of complications after TKA are component loosening and instability. Surgically controllable factors which influence these problems are tibiofemoral misalignment and ligamentous imbalance [4], [8]. While current surgical navigation systems help the surgeon achieve a precise tibiofemoral alignment [5], [7], [12], the ligamentous force balance is still qualitatively assessed through manual trial movements of the limb. By providing an objective and quantitative measurement of the forces acting within the knee, the goal is to help the surgeon improve the accuracy of the ligament balancing procedure, leading to a potentially longer prosthesis lifetime.

A few systems aimed at measuring forces within the knee joint have been reported in the literature. Attfield et al. [1] developed an electronic surgical instrument which contains two plates, one being attached to the tibia, the other supporting the femur. The upper plate acts as a mechanical cantilever in the mediolateral direction and the ligaments are balanced according to the measured inclination of the cantilever, which depends on the moment applied by the soft tissues. A disadvantage of this instrument is the size of its handle, which prevents the patella from being kept in its anatomical place during the measurement. Thus, un- desirable forces are generated by the patella tendon and may lead to a nonoptimal balancing. Wallace et al. [14] used a dual-array electronic pressure transducer (Tekscan, Inc., Boston, MA). The 10\% intrinsic accuracy of the device might be insufficient for such an application and the large number of wires needed by the pressure sensor arrays for the data acquisition requires an additional arthrotomy. Knee joint pressure distribution was also measured intraoperatively with Fuji pressure-sensitive films [13]. However, the accuracy is relatively low and the reading is not available in real-time. In another development, an 
implantable tibial tray was instrumented with four load cells and a passive telemetric transmission system [3], [6], [9]. Besides the long-term postoperative measurements, the device can be used to intraoperatively evaluate the contact forces once the trial prosthesis has been installed. Davy et al. [2] reported a similar concept. Winemaker [15] used a mechanical tensor/balancer device (Stryker Howmedica Osteotonics, Mahwah, NJ) for 83 consecutive TKAs and concluded that this device provides an accurate and reproducible way to measure gap differences and angular asymmetry. However, the disadvantage here is that again, the patella has to be dislocated to perform the measurements.

To overcome these disadvantages, our goal was to develop a small force-sensing device for TKA which simultaneously provides precise, real-time, quantitative measurements with the patella in its anatomical place.

\section{Methods}

\subsection{Modeling}

In this section, a simplified biomechanical model of the knee joint in the coronal plane is presented in order to establish the relationship between the ligamentous balance and the tibiofemoral forces. The model considers the collateral ligament forces $F_{\mathrm{ML}}, F_{\mathrm{LL}}$ and the condyle contact forces acting on the tibia $F_{\mathrm{MC}}, F_{\mathrm{LC}}$ (see Fig. 1). The cruciate ligaments and the patella tendon are neglected since their effect is mainly limited to the sagittal plane. In static conditions, the mechanic equilibrium equations, $\sum_{i} \overrightarrow{F_{l}}=\overrightarrow{0}, \sum_{i} \overrightarrow{M_{l}}=\overrightarrow{0}$, must be satisfied, yielding

$$
\begin{aligned}
& 0=F_{\mathrm{ML}}+F_{\mathrm{LL}}-F_{\mathrm{MC}}-F_{\mathrm{LC}} \\
& 0=x_{\mathrm{ML}} \cdot F_{\mathrm{ML}}-x_{\mathrm{LL}} \cdot F_{\mathrm{LL}}-x_{\mathrm{MC}} \cdot F_{\mathrm{MC}}+x_{\mathrm{LC}} \cdot F_{\mathrm{LC}}
\end{aligned}
$$

where $x_{\mathrm{i}}(i=\mathrm{ML}, \mathrm{LL}, \mathrm{MC}, \mathrm{LC})$ are the lever arms of the different forces measured from the origin placed at $L / 2, L$ being the distance between the collateral ligaments. With the lever arms of the collateral ligaments being equal to $L / 2$, (2) transforms to

$$
M_{\mathrm{net}}=x_{\mathrm{MC}} \cdot F_{\mathrm{MC}}-x_{\mathrm{LC}} \cdot F_{\mathrm{LC}}=L / 2 \cdot\left(F_{\mathrm{ML}}-F_{\mathrm{LL}}\right)
$$

where $M_{\text {net }}$ is the net varus-valgus moment of the collateral ligaments acting on the knee joint. When the tensions in the collateral ligaments are equal $\left(F_{\mathrm{ML}}=F_{\mathrm{LL}}\right)$, the right side of the equation is zero. $M_{\text {net }}$ may therefore be regarded as the parameter characterizing the ligamentous imbalance and reducing it to zero is equivalent to balancing the ligaments. In conclusion, to assess the ligamentous imbalance, not only the amplitude of the contact forces $F_{\mathrm{MC}}, F_{\mathrm{LC}}$ but also their location must be measured.

\subsection{Force Amplitude- and Location-Sensing Device}

The proposed force amplitude- and location-sensing device consists of two sensitive plates, one for each condyle, a tibial base plate, which is fixed by pins, and a set of lateral and medial spaces, which allow the device thickness to be adapted to the patient-specific tibiofemoral gap (see Fig. 2). Thanks to its small thickness $(6 \mathrm{~mm})$, the entire device fits, after an initial tibial precut, inside the knee joint in the tibiofemoral gap with the patella in its anatomical place. Consequently, the ligamentous balance assessment can be carried out at the beginning of the surgery in full extension, at $90^{\circ}$ flexion as well as during passive knee flexions. Performing the assessment of the joint stability at an early stage of the TKA procedure offers the possibility to make a better plan for the femoral cuts and the femoral component rotation. However, since deviations from the plan may occur and thereby alter the knee balance, additional measurements and ligament adjustments may be performed with the trial components in place using a modified version of the measurement system. With this approach, the first stability assessment made at the beginning of the surgery allows better planning of the femoral cuts, whereas the second assessment at the end of the surgery allows fine-tuning of the ligamentous balance. 


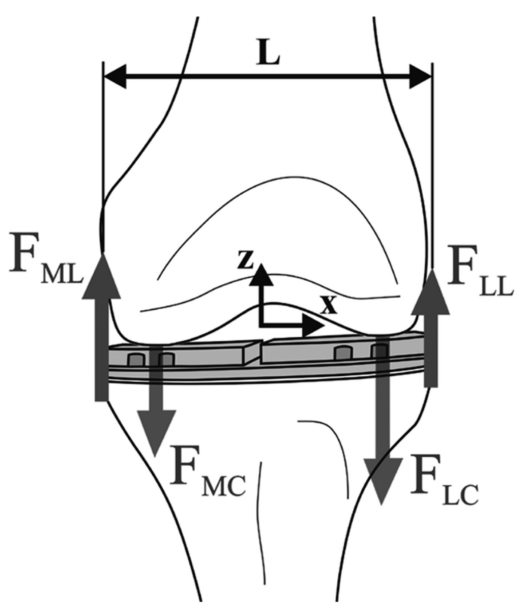

Fig. 1. A simplified two-dimensional biomechanical model of a knee joint in the coronal plane. The forces of the patella tendon and the cruciate ligaments acting mainly in the sagittal plane are neglected; only the collateral ligament forces $\left(F_{\mathrm{ML}}\right.$, $\left.F_{\mathrm{LL}}\right)$ and the contact forces $\left(F_{\mathrm{MC}}, F_{\mathrm{LC}}\right)$ are taken into account.

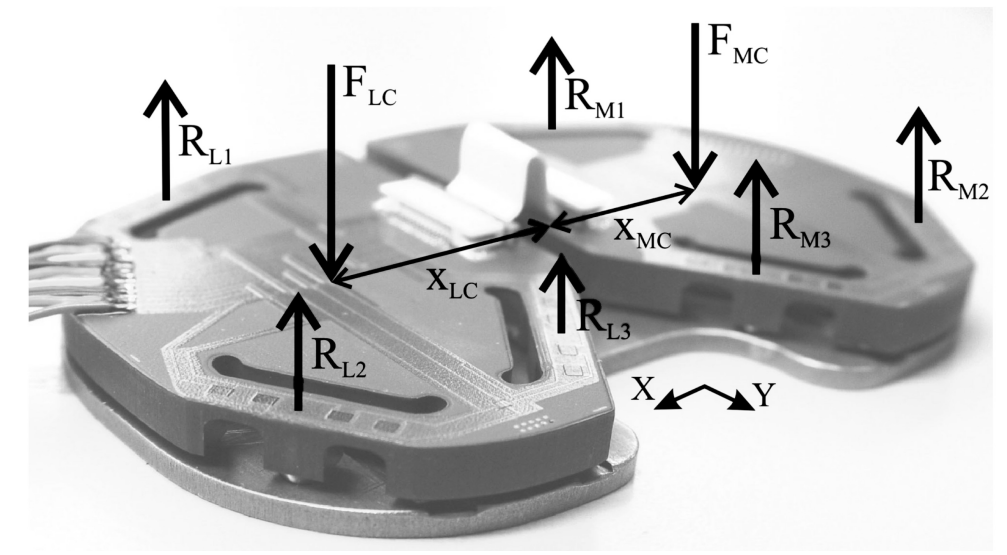

Fig. 2. The device sensitive plates, each containing three deformable bridges instrumented with thick-film piezoresistive sensors. By measuring the reaction forces $\left(R_{\mathrm{ij}}\right)$ through the deformation of the bridges, the amplitude and location of the applied loads $\left(F_{\mathrm{i}}\right)$ can be computed.

Each sensitive plate contains three deformable bridges instrumented with strain gauges (see Fig. 2). The bridges have been designed to remain in the elastic deformation range for loads of $0-500 \mathrm{~N}$, which offers a sufficient safety margin, since passive loads acting in the knee joint during the surgery are expected to vary between 0 and $100 \mathrm{~N}$. Thick-film piezoresistive sensors [10] are used instead of standard bonded metallic strain gauges, which are space consuming and difficult to sterilize. Applying a load in the sensitive area of the device deforms the instrumented bridges proportionally to the amplitude of the reaction forces generated in each bridge pillar (see Fig. 2). The amplitude and location of the applied load can, thus, be determined from the static equilibrium conditions, $\sum_{i} \vec{F}_{l}=\overrightarrow{0}, \sum_{i} \overrightarrow{M_{l}}=\overrightarrow{0}$, which for the medial sensitive plate leads to

$$
\begin{aligned}
& F_{\mathrm{MC}}=R_{\mathrm{M} 1}+R_{\mathrm{M} 2}+R_{\mathrm{M} 3} \\
& x_{\mathrm{MC}}=\left(x_{\mathrm{M} 1} \cdot R_{\mathrm{M} 1}+x_{\mathrm{M} 2} \cdot R_{\mathrm{M} 2}+x_{\mathrm{M} 2} \cdot R_{\mathrm{M} 2}\right) /\left(R_{\mathrm{M} 1}+R_{\mathrm{M} 2}+R_{\mathrm{M} 3}\right) \\
& y_{\mathrm{MC}}=\left(y_{\mathrm{M} 1} \cdot R_{\mathrm{M} 1}+y_{\mathrm{M} 2} \cdot R_{\mathrm{M} 2}+y_{\mathrm{M} 2} \cdot R_{\mathrm{M} 2}\right) /\left(R_{\mathrm{M} 1}+R_{\mathrm{M} 2}+R_{\mathrm{M} 3}\right)
\end{aligned}
$$

where $R_{\mathrm{Mi}}, x_{\mathrm{Mi}}, y_{\mathrm{Mi}}$ are the amplitudes of the reaction forces and the locations of the bridges respectively and $R_{\mathrm{MC}}, x_{\mathrm{MC}}, y_{\mathrm{MC}}$ are the amplitude and location of the applied load respectively. The same (4) hold for the lateral side after replacement of the index M by L. Knowing the amplitude and the location of each contact force, we can compute the net varus-valgus moment $M_{\text {net }}$ of the collateral ligaments.

\subsection{Validation experiments}

1) Following a calibration, the intrinsic accuracy of the device was evaluated by loading the sensitive area with 18 weights ranging from 0 to $500 \mathrm{~N}$, applied at 20 different locations evenly distributed. The positioning of the tip of the mechanism supporting the weights was controlled by a micrometer table. The precision of the weights was $\pm 0.01 \mathrm{~N}$ and the precision of the tip position $\pm 0.001 \mathrm{~mm}$.

2) To test the function of the device in situ, the prototype was implanted into a plastic knee joint equipped with adjustable springs, which simulated the collateral ligaments. The degree of the ligamentous imbalance was controlled by varying the spring tension. Ten levels of mediolateral imbalance were compared to the measurements provided by our device. 
3) The device was finally tested in a cadaver experiment by an experienced surgeon. After a tibial precut of about $6 \mathrm{~mm}$, the device was installed in the knee gap. In order to verify the consistency between the device measurements and the surgeon's perception, the net varus-valgus moment was measured during a ligament release procedure.

\section{Results}

1) The measurements obtained during the accuracy study demonstrated the linearity and the high accuracy of the system (see Fig. 3). The maximum force amplitude and location errors for the 360 measurements in this experiment were $2.6 \mathrm{~N}$ and $0.8 \mathrm{~mm}$, i.e., a relative error of $0.5 \%$ full scale. In the range $0-100 \mathrm{~N}$, the maximum absolute measurement errors were $1.4 \mathrm{~N}$ and 0.6 $\mathrm{mm}$. The error contribution of the mechanical set-up being negligible, these measured deviations characterize the intrinsic accuracy of the device.

2) In the plastic bone experiment, as expected from the laws of mechanics, a linear relationship was measured between the net varus-valgus moment $M_{\text {net }}$ and the applied spring tensions (see Fig. 4). The resulting proportionality factor of $46 \mathrm{~mm}$ corresponded to the physically measured lever arm $(52 \mathrm{~mm})$ with $13 \%$ relative error. This deviation is due to the cumulative effect of the intrinsic accuracy of the device, the difficulty to precisely set the spring tensions and the play in the arm of the spring forces.

3) During the cadaver experiment, the measured contact forces ranged from 40 to $70 \mathrm{~N}$ when no external forces were applied. When the surgeon manually applied varus-valgus loads similar to those that would be applied during a standard TKA, the maximal contact force reached 350$400 \mathrm{~N}$. Thus, the measurement range of the device $(0-500 \mathrm{~N})$ is appropriate. The surgeon performed a medial ligament release procedure guided by the device measurements until the net varus-valgus moment was reduced from an initial imbalance of 1.25 to $0.15 \mathrm{~N} \cdot \mathrm{m}$. The obtained ligamentous balance was qualitatively consistent with the surgeon's perception of a "balanced knee."

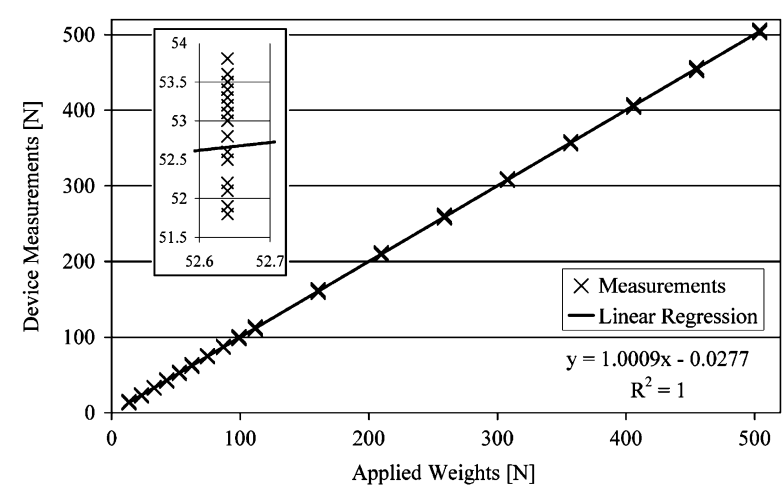

Fig. 3. Comparison between the measured and applied weights to determine the intrinsic device accuracy. The minor deviation of the linear regression slope from the ideal value of 1 demonstrates the linearity and the high accuracy of the system.

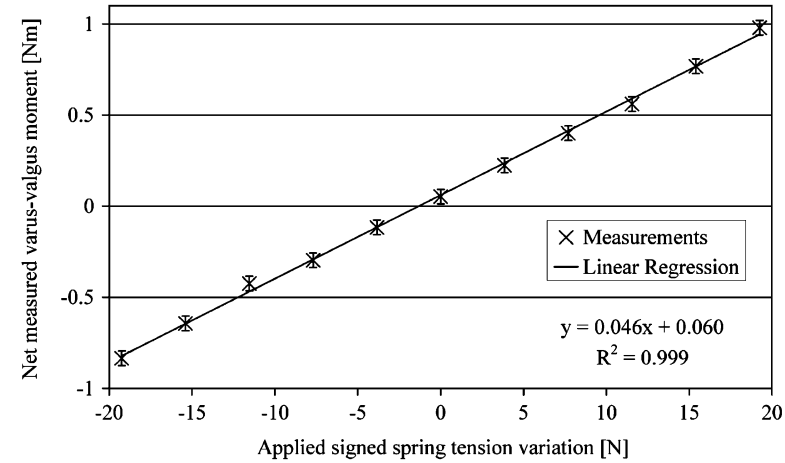

Fig. 4. Comparison between the net varus-valgus moment of the contact forces and the spring tension applied during the plastic knee joint experiment. The expected linear relationship is experimentally verified and the slope of $46 \mathrm{~mm}$ corresponds to the physically measured lever arm $(52 \mathrm{~mm})$ within $13 \%$. Negative (positive) spring tensions refer to the variation of the medial (lateral) spring. 


\section{Conclusion}

The proposed force-sensing device for improved ligament balancing provides not only the amplitude of the tibiofemoral contact forces but also their resulting varus-valgus moment, which is, as shown by the simplified model of the knee, the principal parameter characterizing the collateral ligamentous imbalance. Unlike other systems reported in the literature, the device offers simultaneously the advantage of real-time and precise measurements, keeping the patella in its anatomical place and minimal bone resection, which helps to preserve the joint line. The intrinsic $0.5 \%$ full scale accuracy of the device is sufficient for this application. It is better than that of the Tekscan technology or Fuji pressure-sensitive films (10\%) and comparable to the instrumented tibial tray (1.2\%) [3]. With the plastic bone experiment, the proper function of the device in situ was confirmed. The data acquired during the cadaver experiment demonstrated the appropriateness of the designed measurement range as well as the consistency between the measurements and the surgeon's perception.

The experimental validation of the design described in this paper allows us to conclude that this force amplitude- and location-sensing device has a strong potential to provide useful quantitative information and effective assistance during the ligament balancing procedure in TKA. The clinical benefit of the proposed device will be quantitatively evaluated by comparing both approaches, the standard and the device-assisted, in a series of in vitro and in vivo experiments.

\section{References}

[1] S. F. Attfield, M. Warren-Forward, T. Wilton, and A. Sambatakakis, "Measurement of soft tissue imbalance in total knee arthroplasty using electronic instrumentation,” Med. Eng. Phys., vol. 16, no. 6, pp. 501-505, 1994.

[2] D. T. Davy, G. Kotzar, J. Berilla, and R. H. Brown, "Telemetrized orthopaedic implant work at case western reserve university," in Im- plantable Telemetry in Orthopaedics, G. Bergmann, F. Graichen, and A. Rohlmann, Eds. Berlin, Germany: Forschungsvermittlung der Freien Univ., 1990, pp. 205-219.

[3] D. D. D'Lima, S. Patil, N. Steklov, and C. W. Colwell, Jr., "In vitro measurement of dynamic soft-tissue balance during total knee arthroplasty with an instrumented tibial prosthesis," in Proc. 50th Annu. Meeting Orthopaedic Research Society, San Francisco, CA, 2004, p. 301.

[4] T. K. Fehring and A. L. Valadie, "Knee instability after total knee arthoplasty," Clin. Orthop., vol. 299, pp. 157-162, 1994.

[5] J. Y. Jenny and C. Boeri, "Computer-assisted implantation of total knee prostheses: A case-control comparative study with classical instrumentation,” Comput. Aided Surg., vol. 6, no. 4, pp. 217-220, 2001.

[6] K. R. Kauffman, N. Kovacevic, S. E. Irby, and C. W. Colwell, Jr., "Instrumented implant for measuring tibiofemoral forces,” J. Biomech., vol. 29, no. 5, pp. 667-671, May 1996.

[7] M. Kunz, "Der intraoperative Einsatz von bildfreien, computergestützten Systemen in der Kniechirurgie zur Wiederherstellung der natürlichen Kinematik," Ph.D. thesis, Maurice E. Müller-Institut für Biomechanik, Univ. Bern, Bern, Switzerland, 2003.

[8] J. R. Moreland, "Mechanisms of failure in total knee arthroplasty," Clin. Orthop., vol. 226, pp. 49-64, 1988.

[9] B. A. Morris, D. D. D’Lima, J. Slamin, N. Kovacevic, S. W. Arms, C. P. Townsend, and C. W. Colwell, Jr., "E-Knee: Evolution of the electronic knee prosthesis," J. Bone Joint Surg. Am., pt. 1, vol. 83-A, no. Suppl 2, pp. 62-66, 2001.

[10] M. Prudenziati and B. Morten, "The state of the art in thick-film sensors," Microelectr. J., vol. 23, no. 2, pp. 133-141, 1992.

[11] J.A. Rand, R.T. Trousdale, D.M. Ilstrup, and W.S.Harmsen, "Factors affecting the durability of primary total knee prostheses," J. Bone Joint Surg. Am., vol. 85-A, no. 2, pp. 259-265, 2003.

[12] D.SD. Stulberg, "How accurate is current TKR instrumentation,” Clin. Orthop., vol. 416, pp. 177-184, 2003.

[13] T. Takahashi, Y. Wada, and H. Yamamoto, "Soft-tissue balancing with pressure distribution during total knee arthroplasty,” J. Bone Joint Surg., vol. 79, no. 2, pp. 235-239, 1997.

[14] A. L. Wallace, M. L. Harris, W. R. Walsch, and W. J. Bruce, "Intraoperative assessment of tibiofemoral contact stresses in total knee arthroplasty," J. Arthroplasty, vol. 13, no. 8, pp. 923-927, 1998.

[15] M.J. Winemaker,"Perfect balance in total knee arthroplasty: The elusive compromise," J. Arthroplasty, vol. 17, no. 1 , pp. $2-10,2002$. 